\title{
Le changement de croyances extrêmes
}

du cadre cognitif aux conflits de valeurs

\section{Romy Sauvayre}

\section{CpenEdition}

\section{Journals}

Édition électronique

URL : http://journals.openedition.org/ress/830

DOI : $10.4000 /$ ress.830

ISSN : 1663-4446

\section{Éditeur}

Librairie Droz

\section{Édition imprimée}

Date de publication : 15 octobre 2011

Pagination : 61-82

ISBN : 978-2-600-01551-6

ISSN : 0048-8046

\section{Référence électronique}

Romy Sauvayre, "Le changement de croyances extrêmes », Revue européenne des sciences sociales [En ligne], 49-1 | 2011, mis en ligne le 01 janvier 2015, consulté le 03 mai 2019. URL : http:// journals.openedition.org/ress/830; DOI : 10.4000/ress.830 


\title{
LE CHANGEMENT DE CROYANCES EXTRÊMES DU CADRE COGNITIF AUX CONFLITS DE VALEURS
}

ROMY SAUVAYRE

CNRS / Université de Strasbourg romy.sauvayre@misha.fr

\begin{abstract}
Résumé. Dans le domaine de l'adhésion et de la désadhésion à un mouvement marginal - appelé «secte» par les uns et «Nouveaux Mouvements Religieux» par les autres -, il semble mystérieux de voir un individu accepter des croyances et des pratiques perçues comme irrationnelles, alors que la désadhésion est vue comme un retour «normal» à la raison. Nous nous proposons d'examiner différentes facettes du changement de croyances en abordant successivement le processus d'adhésion, l'apparente «résistance au changement» et la désadhésion à ce type de mouvement et aux croyances qu'il diffuse. Nous verrons que ce processus répond à une logique tout à fait compréhensible au sens wébérien du terme. Une approche cognitive de l'action nous permettra d'explorer les rouages du changement de croyances, au plus près du raisonnement de l'individu, en prenant en considération les modifications qui s'opèrent dans le cadre de pensée ou «cadre cognitif» de l'adepte.
\end{abstract}

Mots-clés: Changement de croyances; cognition; contradiction; valeurs; rationalité; sectes; nouveaux mouvements religieux

\begin{abstract}
In the context of affiliation with or withdrawing from a marginal religious group - known as "cult" by some and as "New Religious Movements" by others - it seems quite a mystery to watch an individual accept beliefs and practices perceived as irrational whereas their withdrawal is perceived as a «normal» return to their senses. We propose to examine different aspects of this change in beliefs as we address successively the affiliation process, the apparent "resistance to change" and the withdrawal from this type of movement and its beliefs. We will see that this process uses a logical rationale that can be perfectly understandable from Weber's standpoint. A cognitive approach will help us explore the workings involved in the change of beliefs, and their effects on the reasoning capacity of the individual, by considering the alterations that happen in the thinking or "cognitive framework" of the follower.
\end{abstract}

Keywords: belief change; disbelief; cognition; contradiction; value; rationality; cult; new religious movements 
INTRODUCTION

Certaines croyances semblent si invraisemblables qu'il n'est pas rare de porter un jugement d'irrationalité sur les personnes qui y adhèrent. C'est notamment ce que peuvent susciter les prédictions annonçant la fin du monde en 2012, l'arrivée prochaine d'une délégation extraterrestre, les prescriptions d’un régime alimentaire spécifique ou de «lumière divine » pour guérir toutes maladies incurables. Le rapport extrême à ces croyances singulières diffusées au sein d'une communauté d'individus a parfois conduit à des comportements extrêmes tels que des assassinats ou des suicides collectifs. Plusieurs événements peuvent ainsi être rapportés non exhaustivement : Charles Manson, alors meneur de la communauté la Famille, prédit l'avènement de la fin du monde et envoya ses plus fervents disciples assassiner en 1969 plusieurs personnes dont Sharon Tate; Jim Jones, fondateur de l'Église du Plein Évangile du Temple du Peuple, mena plus de 900 adeptes vers la mort en 1978; Joseph Di Mambro initia le suicide collectif de 53 membres de l'Ordre du Temple Solaire en 1994.

Ces croyances et ces comportements extrêmes suscitent l'incompréhension des observateurs extérieurs alors que les adeptes qui les endossent les considèrent comme «extraordinaires ». Qu'est-ce qui amène un individu à considérer des croyances défiant le sens commun comme vraies ou probablement vraies? Qu'est-ce qui l'amène à adhérer à des mouvements marginaux appelés «sectes » par les partisans de la manipulation mentale (Abgrall, 1996; Fournier et Monroy, 1999; Singer, 2003) et «Nouveaux Mouvements Religieux» par les sociologues des religions (Barker, 1982 ; Bromley, 1988 ; Champion et Cohen, I999; Dawson, 2006) ? Lors de cette adhésion, le fervent adepte semble incompréhensible, voire irrationnel lorsqu'il se montre imperméable à tout discours s'opposant à ses croyances. Comment expliquer cette absence apparente de perméabilité et de changement de croyances? Enfin, comment expliquer, au moyen de la même théorie, la rupture d’adhésion et d’appartenance à ce mouvement ? 
Nous nous proposons d'examiner différentes facettes du changement de croyances en abordant successivement le processus d’adhésion, l’apparente « résistance au changement » et le processus de désadhésion. Nous verrons que le processus qui mène un individu de l'adhésion à la désadhésion à ce type de mouvement répond à une logique tout à fait appréhendable. Une approche cognitive de l'action nous permettra d'explorer les rouages du changement de croyances, au plus près du raisonnement de l'individu, en prenant en considération les modifications qui s'opèrent dans le cadre de pensée ou «cadre cognitif» de l'adepte.

Pour réaliser cette étude, nous avons eu recours à une population singulière et difficile d’accès pour laquelle les croyances ont occupé une place centrale pendant une période plus ou moins longue de leur parcours biographique. Nous avons donc interrogé, par entretiens biographiques, quarantehuit anciens adeptes de mouvements marginaux de toutes obédiences (philosophique, religieux, guérisseur, spiritualiste, soucoupiste et de développement personnel). Ces adeptes avaient la caractéristique d'avoir adhéré inconditionnellement à des croyances normativement en marge au point que, taxés de fous ou d'irrationnels, ils subirent la moquerie, le rejet social de leurs pairs ou de leur famille'. Ce sont ce que nous appelons les croyances défiant le sens commun. Ces caractéristiques ont rendu plus saillants les mécanismes de la croyance dont nous avons recueilli les changements et les fluctuations au moyen d'échelles de mesure, à savoir les «évaluations du doute »².

I Âgés de 15 à 77 ans pour un âge médian de 47 ans, ces anciens adeptes ont adhéré pendant une durée médiane de huit ans à un ou plusieurs mouvements marginaux. Nous les avons interrogés en Belgique, en France, au Luxembourg et en Suisse de mai à décembre 2008 et disposions alors de 312 heures d'entretiens, pour une durée moyenne de $6 \mathrm{~h} 30$ par enquêté.

2 Ces «évaluations du doute» consistaient à reconstruire la diachronie des doutes vécus par ces adeptes. Chaque doute était alors approfondi pour en obtenir les raisons de son apparition et en évaluer son intensité, l'intensité de l'adhésion à la croyance contredite avant et après la manifestation de ce doute, et enfin l'intensité de l'ébranlement du système de croyances de l'adepte. Au terme de ces «évaluations du doute», nous disposions de plus de 243 doutes évalués comprenant plus de 700 échelles numériques (de «zéro» à «dix») et de plus de 200 échelles qualitatives (de «pas du tout» à «totalement») recueillies auprès de 39 anciens adeptes de notre échantillon. 
En nous appuyant sur ces données empiriques d'une grande richesse, nous nous proposons d'exposer les différentes modalités du changement de croyances, de l'adhésion à la désadhésion, au moyen d'une «approche cognitive » (Boudon, 2008) considérant les raisons des acteurs sociaux comme les causes de leurs actions, et d'une démarche «abductive» (Peirce, I93 I) d'élaboration des hypothèses explicatives.

\section{L’ADHÉSION}

Le processus d'adhésion regroupe deux dimensions aux dynamiques distinctes, mais interreliées: d'une part, l’adhésion au mouvement marginal (les membres et le fondateur), et, d’autre part, l’adhésion aux croyances qu'il diffuse. Les sociologues des religions Merlin Brinkerhoff et Kathryn Burke (1980) distinguèrent déjà dans leurs travaux l'«identification communautaire et la religiosité »; distinction qui fut reprise et approfondie par Stan Albrecht et Howard Bahr (1989). Toutefois, en lieu et place du terme imprécis de «religiosité », l'adhésion aux croyances fera référence ici au processus cognitif à l'œuvre lorsqu'un individu donne son assentiment à une proposition qu'il tient pour vraie ou partiellement vraie (Engel, 2006). Donner son assentiment, comme l'explique Pascal Engel, est un acte volontaire. Celui-ci repose sur de multiples facteurs dont la preuve - médiatisée ou expérientielle - représente l'élément déterminant (Sauvayre, 20I I). L’acceptation successive des croyances proposées amènera à de profonds changements cognitifs, imperceptibles pour l'adepte, à savoir une transformation de sa manière de penser, de raisonner, d'agir, de parler, de se mouvoir, etc. Le nouvel adepte transformera son cadre cognitif en y intégrant progressivement et successivement les croyances descriptives vérifiables, les croyances normatives et enfin les croyances descriptives non vérifiables ${ }^{3}$ proposées par le mouvement marginal.

3 Les croyances descriptives relèvent du vrai ou du faux alors que les croyances normatives relèvent du beau, du bon ou du juste (Boudon, 1999). Les croyances descriptives seront vérifiables lorsque la confrontation au réel (la preuve factuelle) permet d'en valider le contenu et elles seront non vérifiables lorsqu'aucune confrontation au réel ne permet de valider sans équivoque ladite croyance. 
Les termes «cadre cognitif» sont mentionnés dans divers travaux inscrits dans plusieurs disciplines telles que le droit, le management, la psychologie ou la sociologie sans formalisation ou définition tant la réunion de ces termes semble suffisamment évocatrice. Or, les usages relevés sont si divers que nous nous proposons de définir explicitement ce que nous entendons par «cadre cognitif», puis nous en décrirons le processus de transformation. Le cadre cognitif est «l'ensemble des croyances, des représentations et des connaissances qu'un individu mobilise pour toutes opérations de pensée, de raisonnement et d'action. Il est spécifique à chaque individu en ce qu'il se construit au gré des acceptations et rejets de propositions, croyances, connaissances, normes et valeurs auxquelles il est soumis lors de son parcours biographique, et ce, dans un contexte donné. Les éléments mobilisés suite à tout type de stimulations seront fonction de la disponibilité de l'information à l'esprit de l'individu : ce faisant, le cadre cognitif enserre la pensée» (Sauvayre, 2orob, p. 35I). Le cadre cognitif comprend les stocks de connaissances acquis lors de la socialisation primaire et secondaire qu'Alfred Schütz (2008) appelle «schèmes de référence», et il est fonction du «contexte cognitif» (Boudon, 2003) dans lequel évolue l'individu.

Nous entamerons la description du processus de transformation du cadre cognitif à partir des premiers contacts que le futur adepte aura avec les membres de son futur mouvement marginal d'appartenance. Cela ne signifie en rien que l'adepte commence son initiation vierge de toutes croyances, connaissances, représentations, etc.

Sollicité par un proche lui vantant les mérites du groupe et de ses pratiques ou mu par une recherche personnelle, le futur adepte sera initié à la doctrine du mouvement: il assistera à une conférence, lira un livre écrit par son fondateur ou participera à une conversation où seront abordés les bienfaits du mouvement. Ce premier contact aiguisera sa curiosité, mais il restera néanmoins sceptique devant l'univers de croyances défiant le sens commun qui lui est proposé. Au cours d'une dynamique interactionnelle mêlant «coaptation émotionnelle et cognitive» ${ }^{4}$, le futur adepte envisagera la possibilité que ce mouvement, si étrange puisse-t-il lui paraître, peut lui apporter une réponse à tous ses problèmes médicaux, relationnels, 
affectifs ou à toutes ses interrogations (questions existentielles, religieuses, etc.). La curiosité l'emportera alors suffisamment sur le scepticisme pour que le futur adepte accepte d'écouter les propositions qui lui sont faites, voire de les mettre à l'épreuve.

Ainsi, en premier lieu sceptique, le futur adepte débutera son initiation en étant sensible aux incohérences et contradictions susceptibles de lui démontrer que les croyances proposées sont fausses ou probablement fausses. Par exemple: «Utilisez cette technique, tous vos problèmes s'envoleront pour ne laisser place quau bonheur»; «Suivez scrupuleusement cette doctrine et vous développerez des pouvoirs surhumains»; «Suivez ce séminaire et vous guérirez de toutes maladies incurables»; «Suivez notre programme et vous pourrez communiquer avec l'au-delà». Les témoignages des membres du mouvement seront mobilisés comme preuves de la véracité de ces promesses, tout comme le bonheur, la santé et la quiétude qui émanent de l'ensemble des condisciples. Comme tous arborent un sourire rayonnant, le futur adepte ne perçoit aucune contradiction entre les pratiques proposées et les effets attendus de ces pratiques. Ces éléments constituent donc des preuves médiatisées en ce qu'elles sont indirectes. La perception de ces preuves de seconde main sera si convaincante qu'elle produira un doute d'adhésion chez l'adepte. Initialement à l'état de non-adhésion, le futur adepte passe à celui d'adhésion aporétique (la proposition est à la fois probablement vraie et probablement fausse) $)^{5}$. Cette accumulation de preuves médiatisées amène donc le futur adepte à douter de sa non-adhésion.

Schéma I : Les degrés de la croyance et de l'adhésion d'une proposition tenue pour absolument fausse à absolument vraie.

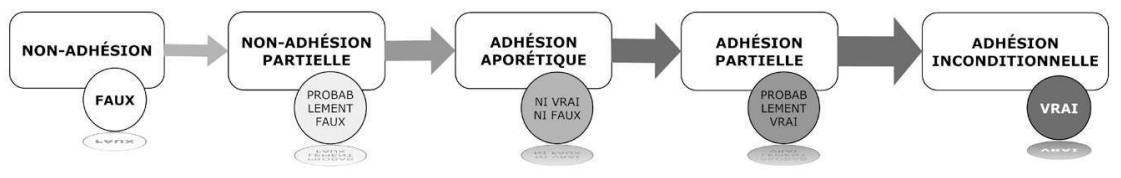

5 L'adhésion aporétique à la croyance se distingue de la «non-croyance» qui se caractérise, selon Willard Quine et Joseph Ullian (1978), par une absence d'adhésion (l'individu n'adhère ni à $p$, ni à non-p). 
Ce doute marque la première transformation importante de son cadre cognitif en ce qu'il ouvre le champ des probables et amène le futur adepte à considérer la proposition comme suffisamment crédible pour la mettre à l'épreuve. Ce faisant, le futur adepte intègre hypothétiquement la proposition à son cadre cognitif et évaluera l'importance des contradictions qu'elle génère, conformément au test de Ramsey (2003), ainsi insérée au cœur de ses croyances antérieures. Or, dès lors qu’il franchit ce pas, il engendre déjà un changement imperceptible, mais durable puisqu'il fera passer artificiellement cette proposition de l'état d'adhésion aporétique à celui d’adhésion partielle pour la mettre à l'épreuve. Il se lance ensuite dans ce que nous avons appelé l'«administration profane de la preuve» (Sauvayre, 20II) et visera à infirmer ou confirmer la validité de la proposition. Là où le chercheur standardisera sa méthode pour mettre à l'épreuve ses hypothèses, le profane analysera les éléments qu'il aura à sa disposition et sera ainsi plus sensible au biais de confirmation (Wason, 1960). Or, lorsqu'il intègre hypothétiquement cette première proposition à son cadre cognitif, nombreuses seront les sources la validant. En effet, dans le processus d'initiation des nouveaux membres d'un mouvement marginal, les premières étapes consistent à présenter des croyances descriptives aisément vérifiables par observation ou par réception des témoignages.

À la suite de cette administration profane de la preuve, l'adepte considère la nouvelle croyance comme probablement vraie. Celle-ci devient rapidement plus disponible à son esprit alors que sa contradiction est enfouie dans les méandres de son cadre cognitif. Le procédé se répétera de sorte que, au gré des acceptations partielles de ces croyances, son cadre cognitif se transforme pour laisser une place de plus en plus importante au cadre de pensée proposé par le mouvement. L'intérêt de l'adepte pour son nouveau mouvement d’appartenance s'accroît. La multiplication de ces adhésions partielles rend disponibles à son esprit ces croyances descriptives lorsqu'il recourra à son cadre cognitif pour raisonner, délibérer ou agir. Cela se traduit par une augmentation du temps consacré au mouvement, un changement de langage et de comportements souvent pointés par les proches du futur adepte, mais imperceptibles aux yeux de l'adepte. En outre, toujours sceptique, mais à la curiosité affûtée, 
il posera de nombreuses questions aux membres du mouvement, lesquelles seront toujours honorées d'une réponse, même si celle-ci n'est pas toujours convaincante ou lui semble peu appréhendable. Mais on lui expliquera alors qu'il comprendra plus tard lorsqu'il aura passé plus de temps dans le mouvement. Par ailleurs, l'assurance et la force de l'argumentation accompagnant le discours des membres du mouvement, souvent composés de paralogismes, contribuent également - au même titre que la preuve médiatisée - à générer des doutes, suivis d'adhésions partielles et à la poursuite du processus de transformation du cadre cognitif du futur adepte.

Nous avons ainsi abordé le parcours de l'adepte de la non-adhésion à l’adhésion partielle amenant à une transformation partielle du cadre cognitif. Un dernier degré d'adhésion reste à atteindre pour que l'adepte évolue vers une adhésion inconditionnelle au mouvement et aux croyances qu'il diffuse et à une transformation complète de son cadre cognitif.

Ce dernier degré d'adhésion sera atteint lorsque l'adepte mettra à l'épreuve les croyances descriptives proposées en les expérimentant par lui-même, sous la sollicitation des membres du mouvement. Devant les réticences du futur adepte, ces membres l'inviteront à faire l'expérience de ce qu'ils avancent. Tout ce qui sera perçu par les différents sens sera commué en informations susceptibles d'être encodées en mémoire pour constituer un stock idiosyncrasique de connaissances subjectives sur le monde. Cette connaissance subjective fournie par les sens de cet individu sera confrontée aux assertions, aux hypothèses et aux croyances qui appelleront à une délibération au terme de laquelle il les tiendra pour fausses, vraies ou probables. Toute expérience personnelle éprouvée subjectivement et individuellement aura alors une valeur de vérité absolue en ce qu'elle est, par définition, de première main et non médiatisée par un tiers. Dès lors que l’adepte fait l'expérience subjective de la véracité de la proposition, il considère qu'il détient une preuve formelle et incontestable qui ne peut souffrir aucune contradiction. Cette acquisition de preuves subjectives amènera alors l'adepte à nourrir une adhésion inconditionnelle et indéfectible à la croyance, ainsi mise à l'épreuve. Dès lors, cette croyance expérientielle sera durablement ancrée dans le cadre cognitif de l'adepte, non comme une 
croyance, mais comme une connaissance fiable, personnelle et subjective sur le monde, puisqu'elle aura été éprouvée comme réelle. Plus encore, la manifestation émotionnelle qui suit cette expérience accréditera, par effet de contagion positive, la doctrine, les membres et le mouvement auquel il appartient depuis peu. L’adepte change alors d'opinion sur le mouvement: de sceptique, il devient un fervent adepte tant il est convaincu d'appartenir à un mouvement hors du commun, susceptible de lui apporter tout ce qu'il désire, et bien plus encore ainsi que l'illustrent les témoignages suivants :

Donc, pour moi, c'était un résultat... tellement inespéré: c'est vraiment au-delà de ce que j'aurais pu imaginer que [ce mouvement] pouvait mapporter. [...] Je découvre ce que ça mapporte là et donc j'ose même pas imaginer la suite! Et donc, ça m’a apporté des choses [...] qui allaient au-delà de ce que javais pu imaginer et découvrir donc forcément c'est extraordinaire. (Bernard, ancien adepte.) ${ }^{6}$

Ce qui devient curieux, c'est qu'il [le fondateur] apporte une réponse à tout. Il apporte une réponse très précise sur ce qui se passe après la mort. La réaction qu'on a eue, c'est de dire qu'on est tombé sur quelque chose de formidable. Parce que lui, c'est vraiment quelqu'un qui sait et on est tombé sur le vrai truc! Et on est tombé sur un trésor. Et c'est tout ce que les membres du mouvement nous disent: «Nous, on a un trésor dans les mains; les autres, ils ne l'ont pas». Parce que non seulement il y a une réponse théorique, mais il y a des pratiques qui peuvent nous conduire à expérimenter ces choses-là. (Jérôme, ancien adepte.)

La preuve expérientielle provoquera donc un basculement sans précédent dans le cadre cognitif. Les éléments transmis par le mouvement et endossés par l'adepte occupaient une place de plus en plus importante dans ledit cadre, mais les croyances antérieures restaient en concurrence avec les nouvelles croyances acquises. Or, ce basculement dans l’adhésion les amenant à considérer le mouvement et ses apports comme «extraordinaires», conduit dès lors les adeptes à considérer comme probablement vraies toutes nouvelles croyances si tant est qu'elles fassent sens pour l'individu. Nul scepticisme ne

6 Afin de garantir l'anonymat des enquêtés rencontrés, nous ne pouvons donner plus de précision sur la nature du mouvement auquel ils ont adhéré. 
semble subsister : puisque tout s'est démontré vrai jusqu'à présent, il est raisonnable de penser que tout sera vrai à l'avenir?.

C'est le côté, on raconte 53 conneries et puis au milieu on va raconter que manger des carottes c'est bon quoi. Et donc à partir du moment où on teste, qu'on mange des carottes, et qu'on se sent bien, on se dit: «ben donc, tout le reste doit être vrai». Il y avait un petit côté comme ça. Il y avait un côté puisque ces techniques sont agréables, aisément accessibles, positives, etc., ça rajoute au fait que certainement, le reste doit être vrai et bon quoi. (Sylvain, ancien adepte.)

Plus encore, un glissement inhérent au processus d'initiation s'opère entre croyances descriptives vérifiables, croyances normatives et croyances descriptives non vérifiables. La doctrine considérée d’une absolue vérité, reliera l'ensemble de ces types de croyances qui relèvent du vrai et du faux tout en étant prescriptives. Alors que les croyances normatives ne peuvent être ni vraies, ni fausses, elles deviennent validables selon les critères du vrai ou du faux en ce qu’elles alimentent et démontrent la véracité des croyances descriptives non vérifiables. Ce faisant, le nouvel adepte se doit d'endosser la totalité de la doctrine qui lui est présentée comme une absolue vérité, une connaissance unique sur le monde qui reste inaccessible au monde scientifique. Il a la chance de faire partie du seul groupe disposant de cette «vérité», lui explique-t-on.

\section{DE LA «RÉSISTANCE AU CHANGEMENT» À LA RÉVISION MINIMALE DES CROYANCES}

Ce glissement entre croyances, connaissances, prescriptions et valeurs mène progressivement à l'hégémonie, dans le cadre cognitif de l'adepte, des modes de pensée et d'agir proposés par le mouvement d’appartenance; ils seront sans cesse réactualisés à l'esprit de l'adepte par les pratiques qu'il se doit de suivre. Le regard que porte l'adepte sur le monde change alors durablement : chaque question trouve une réponse au sein du mouvement et aucun apprentissage antérieur n’a besoin d'être mobilisé ou n'est nécessaire. Il est d'ailleurs invité à se délester de ce bagage encombrant que représente sa vie antérieure. La

7 On retrouve cette dynamique dans l'acceptation d'«idées douteuses, fragiles ou fausses» (Boudon, 1990). 
disponibilité des croyances acquises au sein du mouvement est totale ; c'est en ce sens que le cadre cognitif proposé par le mouvement devient hégémonique.

Par exemple, avant son adhésion inconditionnelle, l'adepte pouvait solliciter la théorie du réchauffement climatique pour expliquer la recrudescence des catastrophes naturelles; après son changement de cadre cognitif, l'adepte considérera que ces catastrophes sont le signe annonciateur de la fin du monde. Il n’aura pas abandonné la théorie du réchauffement climatique, mais celle-ci ne sera plus sollicitée puisque l'idée des signes de la fin du monde est d'une absolue certitude et ne laisse aucun doute sur l'avenir du monde.

L'extrait suivant illustre également ce changement de cadre cognitif, de vision du monde et le passage du degré d’adhésion partielle à celui d’adhésion inconditionnelle :

Avant le changement de cadre cognitif:

[Dans ce livre] il y a toute une relecture comme ça de plein de passages de la Bible, de la Genèse [...], de machin, de truc, même du Nouveau Testament avec à chaque fois des explications de science-fiction quoi. Et puis moi ça m’avait fait chier parce qu'il y avait beaucoup de passages bibliques dans la terminologie biblique. Donc, avec vraiment la façon de parler. Je trouvais ça chiant et sans intérêt quoi. (Sylvain, ancien adepte.)

Après le changement de cadre cognitif:

[Mais] je crois que je l'ai relu [le livre] un ou deux ans après, en fait, mais là, un ou deux ans après, j'étais déjà inféodé à la croyance donc, d'une certaine manière, je l'ai lu avec les yeux du croyant. [...] je le regarde déjà avec des yeux, avec des lunettes particulières quoi. C'est-à-dire, en fait, je refabrique une histoire quel que soit le passage, j'essaie de plaquer le paradigme [du mouvement] dessus et donc ça me semble plutôt intéressant, et donc je suis dans une démarche, comment on pourrait dire, très immodeste en disant: «Ah les connards de croyants qui pensent que ça veut dire ça alors qu'en fait ça veut dire complètement autre chose!» Je crois détenir une vérité. (Sylvain, ancien adepte.)

L'univers de l'adepte est alors totalement déterminé et déterministe, nul doute ou incertitude ne subsistent puisqu'il dispose d'une source de connaissance d’une absolue fiabilité : la doctrine du mouvement. 
C'est ainsi qu'à la suite de la mise à disposition de preuves subjectives expérientielles, l'adepte a adopté des croyances défiant le sens commun, et qu'il a progressivement annihilé tout scepticisme. Tout observateur extérieur pourra tenter de présenter des preuves factuelles, des argumentations solides ou de mettre en place des démentis factuels, aucun doute ou contradiction n'ébranlera le cadre cognitif de l'adepte: l’adepte est imperméable au doute. Cette apparente statique des croyances est perçue comme irrationnelle par un observateur extérieur. Or, dans le cadre de pensée de l’adepte, le processus d’adhésion l'a conduit à considérer la doctrine du mouvement comme relevant d'une absolue vérité et, par opposition, tout ce qui provient de l'extérieur comme absolument faux. Tant que le cadre cognitif endossé au sein du mouvement se montre efficace à répondre à toutes les contradictions qui peuvent se présenter, il ne dispose pas de raisons suffisantes pour faire appel à des éléments concurrents précédemment stockés en mémoire.

Pourtant comme la très grande majorité des adeptes issus de mouvements marginaux ont une activité sociale ou professionnelle hors du mouvement, les sources de contradiction peuvent être nombreuses. Néanmoins, certaines contradictions marqueront plus que d'autres l'adepte et auront des effets distincts sur la dynamique du changement de croyances. Ces contradictions sont de deux types : factuelle et axiologique. Attachons-nous en premier lieu à la contradiction factuelle et à ses effets sur les croyances de l'adepte.

La contradiction factuelle naît d'une opposition entre une croyance et un jugement de fait, à savoir une description objective d'un état du monde. Cette contradiction factuelle oppose un événement objectivement vrai à une croyance subjectivement vraie $e^{8}$ toutes deux ne pouvant être vraies en même temps eu égard au principe de contradiction aristotélicien . La contradiction factuelle se

8 Nous considérons indifféremment les croyances objectivement vraies (dont on peut déterminer sans équivoque la vérité ou la fausseté par l'administration de la preuve) et les croyances subjectivement vraies (considérées comme vraies par l'individu quels que soient les arguments mobilisés pour établir cette vérité).

9 La contradiction factuelle comprend indifféremment des contraires et des contradictoires, car seule la condition de vérité subjective nous intéresse ici. Certes, nous posons sans discussion le principe de contradiction comme valide alors que de nombreux contradicteurs 
manifeste donc lorsqu'un attendu prescrit par un élément de la doctrine ne se concrétise pas («Buvez cette potion, vous guérirez du cancer sans coup férir », mais la rémission ne survient pas) ou lorsqu'une prédiction se révèle objectivement fausse («vous enfanterez un garçon» vous prédit-on, mais vous donnez naissance à une fille). Ce type de contradiction a la particularité de ne pouvoir être mis en cause - l'adepte ne peut que constater et accepter que l'enfant qu'elle vient de mettre au monde est une fille et non un garçon - et constitue une opposition factuelle sans équivoque.

Pourtant la contradiction factuelle aura un effet minimal sur l'adhésion de l'adepte. Si elle amène l'adepte à questionner l'inattendu produit, le cadre cognitif hégémonique pourra, à lui seul, donner du sens à cette contradiction sans qu'il ne se produise de doute dans son esprit. La contradiction ne produit donc, dans un premier temps, aucun changement de croyances lorsqu'elle est médiatisée: le discours d'un proche n'aura que peu d'effet par rapport aux certitudes dont il dispose au sein du mouvement. Comme lors de la phase d’adhésion, ce type d'éléments médiatisés n’a pas d’effet suffisant sur les convictions d'un individu, mais peut toutefois être encodé en mémoire. En revanche, lorsque la contradiction factuelle repose sur des éléments expérientiels (de première main), l'effet produit est plus important et génère un doute. Ce doute le conduit à rechercher les causes de cet inattendu et d'en trouver une explication suffisamment pertinente. Il aura alors recours à ses condisciples, au fondateur et à la doctrine qui restent les personnes les plus éclairées pour lui permettre de comprendre ce qui lui semble être une faille dans le cadre déterministe du mouvement. Une fois que les réponses apportées sont suffisamment pertinentes pour satisfaire son manque de compréhension, le doute se dissipe, mais laisse une trace mnésique marquante qui caractérise le moment où le cadre cognitif endossé s'est montré défectueux. Toutefois, les croyances qui auront été directement contredites - alimentées par une expérience de première main - seront révisées sans produire de rupture d’adhésion ou de changement notable du cadre cognitif. L'extrait suivant exprime bien cet 
effritement partiel des croyances: «C'était pas tout le bloc [des croyances] qui s'effritait, c'était juste un petit bout de béton qui est tombé!» (Didier, ancien adepte).

Le schéma 2 ci-après issu de l'évaluation du doute de Laurianne montre que les deux premiers doutes importants qu’elle a vécus au cours de son adhésion n'ont eu aucun effet sur l'intensité de son adhésion, alors que le troisième a provoqué un changement minimal ${ }^{1 \circ}$. Convaincue que son mouvement d'appartenance peut guérir toutes les maladies, elle considère que la médecine est inutile. Or, elle dut recourir à leurs soins, ce qui provoquera une importante contradiction qui eut pour conséquence le changement de cette croyance visà-vis de la médecine. Toutefois, elle ne remit pas en question le fait que son mouvement n’ait pas pu la guérir, et ce, pour de bonnes raisons ${ }^{11}$.

Schéma 2: Courbe de désadhésion de Laurianne ${ }^{12}$

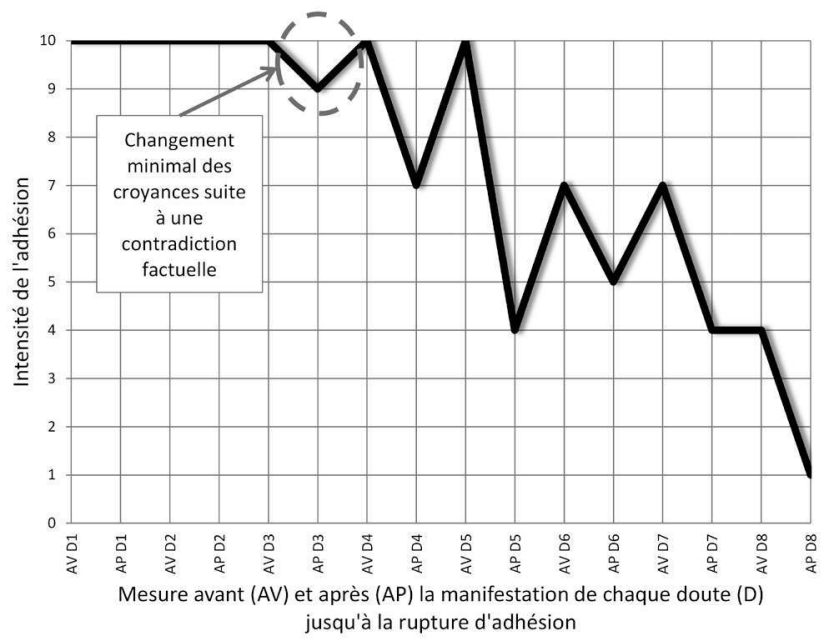

10 Conformément à la «maxime de mutilation minimale» énoncée par Quine (1973) ou de «changement minimum » proposé par le paradigme AGM (Alchourrón, Gärdenfors et Makinson, 1985), un individu n'abandonnera que les croyances qui conduisent à une restructuration minimale du système de croyances.

II Voir Sauvayre (2010a) pour approfondir.

12 Ce graphique issu des «évaluations du doute» ne représente pas les données temporelles entre chaque doute: il peut s'écouler d'un doute à l'autre une durée comprise entre quelques heures et une quinzaine d'années. 
L'absence de rupture d’appartenance à la suite de telles contradictions trouve également un appui empirique dans le fait quaucune rupture d’adhésion à un mouvement marginal ne s'est produite à la suite du premier ou du deuxième doute important vécu par les adeptes (cf. schéma 3 ci-dessous).

Schéma 3 : Répartition du nombre de doutes ressentis par les adeptes précédant la rupture d’appartenance à un mouvement marginal ${ }^{13}$.

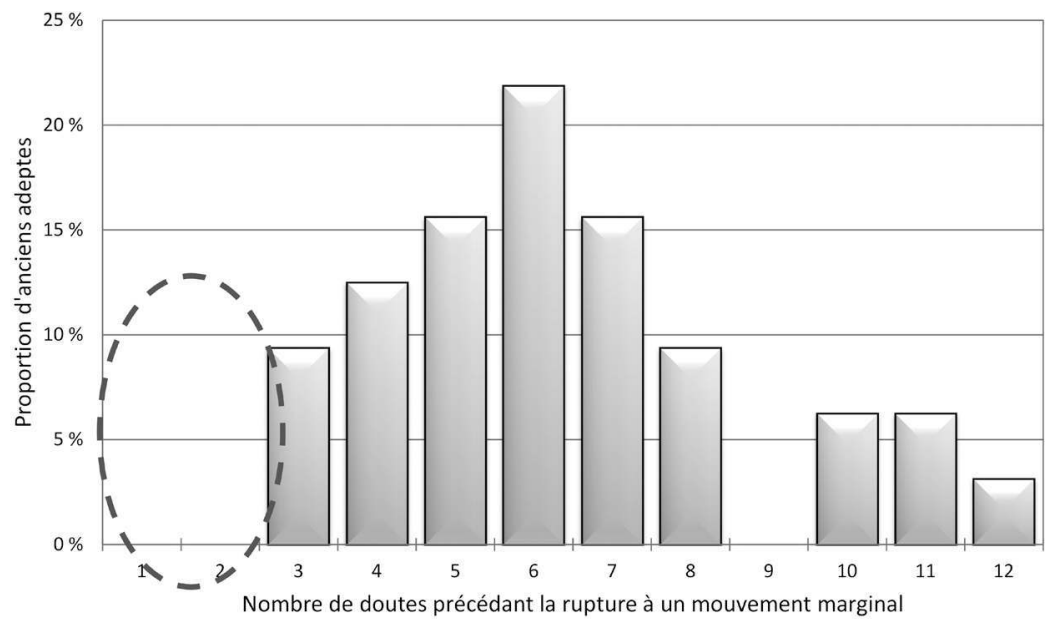

Si l'on pouvait attendre une rupture d'appartenance dès le premier doute important ressenti, les données recueillies montrent que l'hégémonie du cadre cognitif se maintient à la suite des deux premières contradictions vécues, si intenses soient-elles.

13 Les doutes recensés et évalués sont ceux qui ont le plus fort impact (émotionnel et cognitif) sur l'adepte à partir de l'adhésion inconditionnelle jusqu'à l'abandon des croyances acceptées au sein du mouvement. Pour certains, le dernier doute provoque à la fois la rupture d'appartenance au mouvement et la rupture des croyances, et pour d'autres, la rupture d'appartenance précède la rupture des croyances principales acceptées. Ainsi, ce dernier doute peut représenter le départ physique du mouvement ou l'abandon de croyances au fort «enracinement épistémique» après la sortie, pour reprendre les termes de Peter Gärdenfors et David Makinson (1988). 
Par ailleurs, si les contradictions factuelles peuvent produire une fluctuation dans l'intensité de l'adhésion, elles n'engendrent que rarement (29\% des cas) une rupture d'adhésion incluant l'abandon des croyances et le départ du mouvement d'appartenance. C'est ainsi que le démenti d'une prédiction, perçu par l'observateur comme une preuve indiscutable permettant de produire une rupture d’adhésion, ne provoque pas systématiquement la défection des membres de mouvements marginaux. En outre, nous pouvons également constater, avec le schéma 3, que la rupture d’adhésion se produira en moyenne au terme du sixième doute. Le processus de changement de croyances et de cadre cognitif est donc un processus graduel et fluctuant qui voit l'intensité de l'adhésion décroître progressivement jusqu'à la rupture complète (cf. schéma 2). Mais si la contradiction factuelle a un effet minimal sur les croyances au point de laisser voir à l'observateur extérieur une apparente statique de l'adhésion, comment expliquer cette dynamique du changement de croyances qui conduit le fervent adepte à la rupture d’adhésion?

\section{DÉSADHÉSION ET CHANGEMENT DE CADRE COGNITIF}

Les ressorts du changement de croyances sont donc à explorer auprès d'un autre type de contradiction : la contradiction axiologique. La contradiction axiologique naît d'une opposition entre une croyance normative relevant du bon, du juste et du beau (Boudon, 1999) et d'un jugement de valeur contredisant cette croyance, à savoir une évaluation subjective d'un fait, d'un événement, d'un comportement ou d'un acte de discours. La contradiction axiologique naissant d'une telle évaluation peut se manifester lorsque les croyances et valeurs collectives intériorisées par l'adepte entrent en contradiction, soit avec un jugement de valeur porté sur un tiers (un condisciple ou le fondateur de la croyance), soit avec les valeurs individuelles de l'adepte. Les jugements de valeur qui marqueront plus particulièrement la contradiction axiologique portent généralement sur le bien et ses déclinaisons (le juste et l'utile). Ainsi les valeurs mises en cause ont souvent une dimension normative: «il se doit d'être solidaire, dévoué, etc. ». 
Rappelons que le processus d'initiation d'un nouvel adepte comprend l'acceptation de croyances descriptives vérifiables, non vérifiables et des croyances normatives. Ces croyances, diffusées comme des savoirs d'une absolue vérité, sont progressivement interreliées et perçues comme relevant des critères du vrai et du faux. Lors de la phase d’adhésion, cela a permis au futur adepte d’accepter des croyances descriptives non vérifiables comme s'il disposait de preuves expérientielles de première main ; l'émotion et les sensations étant considérées comme validant la véracité de ces croyances non vérifiables. Les prescriptions, les valeurs, les pratiques et les croyances sont donc profondément reliées dans le cadre cognitif de l'adepte. Or, lorsqu'une contradiction axiologique s'oppose aux valeurs centrales de l'adepte, celle-ci est considérée comme inacceptable et injustifiable. La contradiction se propagera ensuite à l'ensemble de la doctrine acquise au sein du mouvement. Le choc émotionnel occasionné par ce conflit de valeurs migre vers un vif sentiment d'indignation, de révolte ou de colère qui conduira cet adepte à remettre en question, pour la première fois de son parcours, son appartenance au mouvement. La contradiction axiologique se propage ainsi à l'ensemble du cadre cognitif: nul changement minimal ne permet de résorber le doute ainsi produit en ce que le cadre de pensée (doctrine, prescription, etc.) diffusé par le mouvement reste impuissant à expliquer de manière suffisamment convaincante la contradiction axiologique. La contradiction provoque un basculement dans son cadre cognitif. Ce doute de basculement, initié dans $73 \%$ des cas par un conflit de valeurs, survient le plus souvent à la suite du troisième doute. Les éléments transmis par le mouvement ne sont alors plus considérés comme les seuls à même à fournir des explications sur le monde. L’absolue vérité qui entourait le mouvement et la doctrine s'effrite. L'adepte met alors en concurrence ses stocks de connaissances passées qu'il réactualise au sein de son cadre cognitif. Les éléments acquis au sein du mouvement occupaient jusqu'alors l'ensemble de ses processus mentaux, mais à la suite de ce basculement, le changement de cadre cognitif est tel que ceux-ci perdent de leur hégémonie. L’adepte retrouve son scepticisme et s'engage dans une démarche plus critique au cours de laquelle, il sera plus attentif aux contradictions. 
Progressivement, l'adepte remettra en question ses croyances et son adhésion. Chaque nouvelle contradiction vécue constituera autant d'éléments en faveur de la remise en cause complète de son appartenance.

Son cadre cognitif continue néanmoins à changer au point que la vision du monde acquise au sein du mouvement s'effrite et laisse entrevoir davantage de contradictions. L’adepte accepte de considérer comme potentiellement fausses des croyances qu'il avait pleinement endossées au sein du mouvement. Dans une dynamique inverse au processus d’adhésion, il considère hypothétiquement que la proposition non-p soit vraie afin de la mettre à l'épreuve des faits. C'est ce qu'illustre l'extrait suivant:

Il y a eu des étapes en fait. Après être certain de tous ces mensonges [du fondateur] et après avoir relu les bouquins et m'être aperçu que dans les bouquins, il y avait des incohérences monumentales que je n'avais même pas vues et qui d'un seul coup parce que j’avais accepté de remettre en doute la probité du gourou et que j'avais compris qu'il pouvait être menteur. Donc, j'ai relu le bouquin en me disant: «Et si c'était un menteur?». Et donc d'une certaine manière, j’ai trouvé toutes les contradictions, les incohérences qu'il y avait dedans. (Sylvain, ancien adepte.)

Comme l'exprime cet extrait, à mesure que l'adepte déconstruit le cadre de pensée proposé par le mouvement en interrogeant chaque croyance endossée, le regard qu'il porte sur le mouvement change progressivement. Le changement de son cadre cognitif l'amène alors à être plus attentif à des contradictions qu'il ne percevait pas comme telles auparavant.

À la suite de ce basculement, les contradictions se commuant en doute deviennent donc plus nombreuses et s'enchaînent rapidement: du doute de basculement ( $3^{\mathrm{e}}$ doute), au doute de rupture (6 doute), l'adepte passe de l'état d’adhésion aporétique à celui de non-adhésion partielle (cf. schéma I). Changeant de vision du monde, l'adepte se lance dans une «rétroanalyse » de l'ensemble des doutes importants qui a marqué son parcours. Alors quauparavant ces contradictions avaient trouvé une réponse satisfaisante dans les explications diffusées par le mouvement, à présent, l'ensemble de ces éléments est susceptible d'être considéré comme faux. Ladepte réanalyse donc ces contradictions et recherche 
des hypothèses explicatives concurrentes. La doctrine du mouvement se montre plus que jamais impuissante à donner du sens aux contradictions soulevées.

Enfin, la dernière contradiction, le doute de rupture, survenant le plus souvent

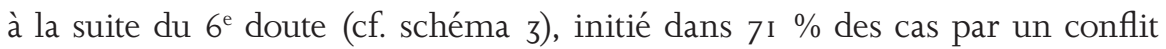
de valeurs, voit l'adepte passer de l'état de non-adhésion partielle à celui de non-adhésion. Avant cela, il doutait encore de ses propres doutes ce qui le plongeait souvent dans un état aporétique, mais avec cette dernière contradiction, la certitude se fait jour et plus aucun doute ne subsiste. Un déclic se produit à l'esprit de l'adepte. Cela se traduit par la nécessité de quitter le mouvement et de rompre avec les croyances acquises, ainsi que l'illustre l'extrait suivant:

Bon là, j'étais déjà plus que sûr d'être sûr d'être sûr! Mais bon quand je l'ai vu [le fondateur] raconter des conneries à l'opposé de l'autre, [...] c'est pas bon et que à chaque fois, ça faisait plusieurs fois, comme ça, qu'il y avait des malentendus. Donc, là c'était le déclic total quoi! Là, je me suis dit c'est j’ai, j’ai plus aucun doute là! C'est du délire complet! Je suis un extraterrestre dans cet univers, moi, je n’ai rien à foutre là-dedans! Là, ça a été le... Ça n’a pas été le déclic amorceur [le doute de basculement], ça a été le déclic qui conclut, qui met un terme [le doute de rupture]. (Didier, ancien adepte.)

À la suite de cette rupture d'adhésion, l'adepte quittera physiquement le mouvement. Mais même s'il y a eu un basculement dans son cadre cognitif et que de nombreuses croyances sont passées de l'état de vrai à celui de faux, l'adepte maintiendra celles, acquises au sein du mouvement, pour lesquelles il dispose de preuves expérientielles alors que d'autres auront un statut aporétique. Il s'ensuit donc une longue période au cours de laquelle l'adepte analysera l'ensemble des croyances acceptées au sein du mouvement, afin de s'en détacher lorsque cela est possible ou de les abandonner.

\section{CONCLUSION}

Le changement de croyances au sein de mouvements marginaux peut sembler relever de l'irrationnel dans la mesure où leurs membres adhèrent à des croyances défiant l'entendement, majoritairement considérées comme fausses au sein de la société dans laquelle ils évoluent par ailleurs. Or, il apparaît 
que le processus d'adhésion et de désadhésion répond à une dynamique tout à fait appréhendable puisque l'adepte accepte progressivement et graduellement des propositions qu'il considérera comme vraies, et ce, pour de bonnes raisons. Ce sera l'accumulation de ces microchangements de croyances - les croyances proposées par le mouvement prenant le pas sur les croyances antérieures - qui provoquera le changement du cadre cognitif de l'adepte. Bien que ses proches perçoivent ces changements qui se traduisent dans le langage et les actions de l'adepte, ce dernier ne les percevra pas avec autant de clarté dans la mesure où il ne considère que l'acceptation ou le rejet de nouvelles croyances les unes après les autres. Comme il adopte une démarche sceptique et critique, l'acceptation du changement de croyances ne se réalise que s'il dispose d'éléments suffisamment convaincants (preuves médiatisées et expérientielles). Par conséquent, il éprouve le sentiment d'être pleinement acteur de son adhésion et de sa désadhésion. Toutefois, ce processus d'adhésion l'amènera à des modifications durables au sein de son cadre cognitif.

La dynamique de changement de croyances est marquée par des basculements qui, dans un premier temps, amènent l'adepte à endosser pleinement la vision du monde du mouvement: il mobilise exclusivement, les éléments transmis par ses membres et son fondateur. Le degré de certitude diffusé au sein du groupe l'amène à évoluer dans un contexte déterminé où l'ensemble des prédictions est considéré comme absolument vrai. Cela explique alors l’apparente «résistance au changement» dont peuvent faire preuve les membres de mouvements marginaux. Or, les changements de croyances sont nombreux, même s'ils sont peu perceptibles aux yeux de l'observateur extérieur. Cette apparente statique des croyances volera en éclat dès lors que l'adepte percevra des contradictions axiologiques.

En effet, le changement de cadre cognitif est un processus dynamique qui recouvre une dimension graduelle dans l'adhésion et la désadhésion à des croyances puisque six doutes sont nécessaires avant que ne se produise la rupture totale d’adhésion. Mais ce processus a également une dimension plus soudaine et brutale lorsque survient un conflit de valeur indépassable qui marque des changements majeurs dans le cadre cognitif de l'adepte. Ce faisant, 
le changement de croyances et la rupture d'appartenance à un mouvement marginal trouvent un élément déclencheur dans les conflits de valeurs ou contradictions axiologiques. Le conflit de valeur a donc une influence majeure et irrévocable sur le système de croyances d'un individu en ce qu'il se diffuse à l'ensemble de son système de croyances pour en provoquer son éclatement et accélérer la rupture d’appartenance au mouvement.

\section{BIBLIOGRAPHIE}

ABGRALL J.-M., 1996, La Mécanique des sectes, Paris, Payot \& Rivages.

ALBRECHT S. L. et BAHR H. M., 1989, Strangers Once More: Patterns of

Disaffiliation from Mormonism, in «Journal for the Scientific Study of Religion», vol. 28, n², p. $180-200$.

ALCHOURRÓN C. E., GÄRDENFORS P. et MAKINSON D., 1985, On the Logic of Theory Change: Partial Meet Contraction and Revision Functions, in «The Journal of Symbolic Logic», vol. 50, n², p. 510-530.

BARKER E., 1982, New religious movements: a perspective for understanding society, New York, Edwin Mellen Press.

BOUDON R., 1990, L'Art de se persuader des idées douteuses, fragiles ou fausses, Paris, Fayard.

-, 1999, La Rationalité axiologique, in Boudon R., Le Sens des valeurs, Paris, PUF, p. 137-203.

-, 2003, Raison, bonnes raisons, Paris, PUF.

-, 2008, Le Relativisme, Paris, PUF.

BRINKERHOFF M. B. et BURKE K. L., 1980, "Disaffiliation: Some Notes on 'Falling from the Faith', in Sociological Analysis, vol. 4I, no I, p. 4l-54.

BROMLEY D. G. (dir.), 1988, Falling from the faith: causes and consequences of religious apostasy, Newbury Park, Sage.

CHAMPION F. et COHEN M., 1999, Sectes et Démocratie, Paris, Seuil.

DAWSON L., 2006, Comprehending cults: the sociology of new religious movements, Don Mills, Oxford University Press. 
ENGEL P., 2006, Croyance individuelle, in Mesure S. et Savidan P. (dir.), Dictionnaire des sciences humaines, Paris, PUF, p. 223-225.

FOURNIER A. et MONROY M., 1999, La Dérive sectaire, Paris, PUF.

GÄRDENFORS P. et MAKINSON D., 1988, Revisions of Knowledge Systems Using Epistemic Entrenchment, in Proceedings of the 2nd Conference on Theoretical Aspects of Reasoning about Knowledge, Sans Francisco, Morgan Kaufmann Publishers Inc., p. 83-95.

LUKASIEWICZ J., 2000, Du principe de contradiction chez Aristote, Paris, L'Éclat.

PEIRCE C. S., 1931, Collected papers of Charles Sanders Peirce, Cambridge, Harvard University Press.

PRIEST G., 1985, Contradiction, Belief and Rationality, in «Proceedings of the Aristotelian Society», vol. 86, p. 99-116.

QUINE W. V. O. et ULLIAN J. S., 1978, The Web of belief, New York, Random House.

- 1973, Méthodes de logique, Paris, Armand Colin.

RAMSEY F. P., 2003, Logique, philosophie et probabilités, Paris, Vrin.

SAUVAYRE R., 2010a, Contradictions factuelles, doutes et rupture des croyances défiant le sens commun: une dynamique contre-intuitive, in Guy B. (dir.), Ateliers sur la contradiction. Nouvelle force de développement en science et société, Paris, Presses de l'École des Mines, p. 303-312.

-, 20l0b, Le Processus d'abandon des croyances défiant le sens commun, thèse: sociologie, Université de Strasbourg.

-, 20II, La Croyance à l'épreuve: une dialectique émotionnelle et cognitive, in Aden J., Grimshaw T., et Penz H. (dir.), Enseigner les langues-cultures à l'ère de la complexité: approches interdisciplinaires pour un monde en reliance, Bruxelles, P.I.E. Peter Lang, p. 121-134.

SCHÜTZ A., 2008, Le Chercheur et le Quotidien, Paris, Klincksieck.

SINGER M. T., 2003, Cults in Our Midst: The Continuing Fight Against Their Hidden Menace, Sans Francisco, Jossey-Bass.

WASON P. C., 1960, On the failure to eliminate hypotheses in a conceptual task, in «Quarterly Journal of Experimental Psychology», vol. 12, n³, p. 129-140. 